\title{
La trampa de la incidencia: el caso del activismo juvenil ecologista en Catalunya
}

\author{
The Advocacy Trap: the case of youth ecologist activism in Catalunya
}

\author{
Ariadna Romans i Torrent \\ Universidad Pompeu Fabra | Carrer de Ramon Trias Fargas, 25, 27, 08005 Barcelona | España | \\ https://orcid.org/0000-0002-7095-5852 | ariadna2402@gmail.com
}

Fechas | Recepción: 27/09/2021 | Aceptación: 14/12/2021

\section{Resumen}

Los movimientos juveniles ecologistas han tomado una deriva diferente en los últimos años, sobre todo después de la aparición de la joven activista Greta Thunberg. La pandemia del coronavirus y su posterior crisis, junto al fenómeno de la digitalización están contribuyendo a la formación de una nueva tendencia dentro del activismo, que algunos autores han definido como nuevos movimientos sociales, NMS (von Kardorff, 2019). Este nuevo tipo de activismo (y también otros movimientos sociales) topa con un reto que es conocido como la trampa de la incidencia, que es la situación donde los jóvenes activistas ecologistas se encuentran con sentimientos de frustración, decepción o desesperación ante su incapacidad de trasladar de forma directa sus demandas a las instituciones que, o bien no las toman en serio, o bien las utilizan para propósitos distintos de los reclamados por parte de los activistas. La trampa puede resultar en reacciones como el abandono del activismo por parte de los jóvenes o el agotamiento de los movimientos. Este artículo tiene tres ambiciones. En primer lugar, esbozar las principales claves de este nuevo activismo juvenil ecologista. En segundo lugar, presentar el principal reto con el que se encuentra en el momento presente, que este artículo ha descrito como "la trampa de la

\section{Abstract}

The environmental youth movements have taken a different drift in recent years, especially after the emergence of the young activist Greta Thunberg. The coronavirus pandemic and its subsequent crisis, together with the phenomenon of digitization, are contributing to the formation of a new trend within activism, which many have defined as new social movements, NMS (von Kardorff, 2019). This new type of activism (and also other social movements) runs up against a challenge that is known as the advocacy trap, which is the situation where young environmental activists run into feelings of frustration, disappointment or despair at their inability to move from directly shape their demands to institutions that either do not take their demands seriously or use them for purposes other than those claimed by activists. The trap can result in reactions such as the abandonment of activism by young people or the exhaustion of movements. This article has three ambitions. First, outline the main keys to this new environmental youth activism. Second, present the main challenge you are facing right now, which this article has described as the advocacy trap. Third, empirically demonstrate from a set of semistructured interviews the effects of this advocacy trap on environmental youth activism, focusing on the main consequences it has on the 
incidencia". En tercer lugar, demostrar empíricamente a partir de un conjunto de entrevistas semiestructuradas los efectos de esta "trampa de la incidencia" en el activismo juvenil ecologista catalán, centrándose las principales consecuencias que tiene sobre el estado emocional de sus integrantes.

Palabras clave: activismo, juventud, ecologismo, incidencia, frustración. emotional state of its members.

Keywords: activism, youth, ecologism,
advocacy, frustration.

\section{INTRODUCCIÓN}

Desde el inicio de la Revolución Industrial el mundo ha sufrido los efectos de una transformación radical en cada aspecto de la política, la sociedad y el sistema económico. A través de estos cambios han surgido movimientos antisistema de protesta e incidencia que han querido transformar este nuevo sistema en otro más justo. Por esta razón, movimientos como el movimiento laborista, las luchas por los derechos civiles o los movimientos ecologistas han emergido desde el siglo XIX para impulsar este cambio de paradigma. Durante el siglo XX numerosos grupos organizados desde los márgenes del sistema han dirigido sus causas a romper la mercantilización de la vida. Las calles han sido uno de los pocos espacios que ha sobrevivido este proceso de mercantilización total, deviniendo uno de los espacios más libres de nuestras sociedades donde los activistas han centrado gran parte de sus acciones. Los cambios esperados en las campañas y repertorios han estado teniendo lugar, con toda probabilidad, desde el final del siglo veinte (Tilly, 2006). Sin embargo, con el nuevo escenario digital, hemos visto el inicio de una nueva forma de activismo.

Si el siglo XIX fue el siglo de la lucha por las condiciones laborales y el siglo XX el de la lucha por los derechos personales y sociales, el siglo XXI será el siglo para el bienestar planetario. Este nuevo siglo se presenta como una intersección de las dos anteriores luchas, donde los derechos laborales y sociales se unen para luchar por otra causa mayor: la lucha contra el cambio climático. La creciente conciencia sobre el cambio climático genera una gran oportunidad para que el activismo juvenil recupere su espacio y presencia en la arena pública. Después de las Primaveras Árabes y el fenómeno Greta Thunberg (Díaz Pérez, 2020), sumado al impacto en nuestras vidas de la crisis del coronavirus, el activismo juvenil, y especialmente el activismo juvenil ecologista, ha reorientado su estrategia para continuar aunando sus luchas y causas sociales. Las nuevas formas de este nuevo tipo de activismo ya llevan años consolidándose. La pandemia del coronavirus y su posterior crisis, sin embargo, abre algunas nuevas posibilidades en el camino.

Recientemente, tendencias de la globalización como el acceso a internet o los vuelos low cost, así como la importancia de la lucha contra el cambio climático han propiciado lo que se conoce como nuevos movimientos sociales (NMS) (von Kardorff, 2019). En alguna ocasión, como en el caso de Juris (2012), también se ha hablado de novísimos movimientos sociales para hacer referencia a estos característicos movimientos sociales. Estos movimientos, que se explicarán más adelante, son la nueva oleada de movimientos ambientales que, con nuevas herramientas tecnológicas y con nuevos valores sociales y colectivos, han iniciado un nuevo tipo de protesta civil en los últimos años. Este trabajo sigue una línea de estudios sobre activismo 
medioambiental (p.ej.: Demaria et al., 2013; Keck y Sikkink, 2014; Kirshner, 2015; O’Brien et al.,2018; Jenkins et al., 2018; Ospina Peralta et al., 2019; Kühne, 2019; Hernández-Ballesteros, 2020; Noguera, Cammarota y Ginwright, 2013), que conforma el marco teórico-conceptual en el que se ha realizado esta investigación.

Este artículo tiene tres objetivos. En primer lugar, esbozar las principales claves de este nuevo activismo juvenil ecologista. En segundo lugar, presentar el principal reto con el que se encuentra en el momento presente, que este artículo ha descrito como "la trampa de la incidencia". En tercer lugar, analizar empíricamente a partir de un conjunto de entrevistas semiestructuradas los efectos de esta "trampa de la incidencia" en el activismo juvenil ecologista, centrándose las principales consecuencias que tiene sobre el estado emocional de sus integrantes.

\section{REVISIÓN TEÓRICA Y ANÁLISIS DE SITUACIÓN}

Si bien los movimientos verdes emergieron en su gran mayoría en la segunda mitad del siglo XX, en los años recientes han vuelto a emerger por diferentes razones, entre ellas la aparición de la activista Greta Thunberg, en un formato renovado impulsado mayoritariamente por la conocida como Generación Z. Su conciencia por la emergencia climática y el peligro de la pérdida de biodiversidad que está sufriendo el planeta, sumado a el hecho de ser nativos digitales, han conformado la naturaleza de un movimiento centrado en resolver la relación nociva entre humanidad y naturaleza.

La generación $Z$ ha crecido en una época de inseguridad en el ámbito laboral y de crisis económica, lo cual los ha forzado a ser autosuficientes y autodidactas. Además, se trata de individuos que, en las sociedades occidentales, han sido educados en la igualdad y la diversidad racial y cultural, propiciando una mayor implicación en temas como los derechos humanos, la sostenibilidad y el cuidado del medio ambiente. Esto ha derivado en ser una generación con una mayor tasa de activismo social (Díaz Pérez et al., 2021).

Los llamados centennials o generación Z son los jóvenes que han nacido con esta percepción. Nacidos entre 1997 y 2012, se caracterizan por varias cuestiones, pero sobre todo por su audacia, su efectividad y simpleza en los mensajes que ha logrado cautivar a los más grandes sectores políticos, empresariales y sociales (Sabherwal et al., 2021). La emergencia climática una de las principales preocupaciones a futuro de la ciudadanía global. En sondeos de People's Climate Vote 2021 (Flynn et al., 2021), una de las encuestas climáticas más grandes realizadas hasta la fecha, el $64 \%$ reconoce el cambio climático como una emergencia global.

Este movimiento experimentó un crecimiento sin precedentes en el año 2019 con la aparición de la activista Greta Thunberg y el nacimiento de Fridays for Future (Wahlström et al., (2019). Según los datos de Wallis y Loy (2021), la participación acumulada a nivel mundial en este movimiento en 2019 fue de 12,8 millones de personas, 2,3 de las cuales participaron en manifestaciones en el mes de marzo y 7,3 millones durante la semana de la huelga climática del mes de septiembre alrededor de la Cumbre para la Acción Climática celebrada en Nueva York, y 1,2 millones en la Marcha por el Clima con motivo de la COP25 en diciembre de 2020 en Madrid (Díaz Pérez, 2020).

Un aspecto importante de estos activistas es que, a diferencia de los movimientos sociales anteriores, este está liderado por personas muy jóvenes. La llamada generación Z es la que 
está liderando este nuevo camino, y parece ser provocada por la sensación de urgencia por mitigar el cambio climático. Como afirmó la socióloga de la Universidad de Maryland, Dana Fisher, en la revista Nature (2019): "Los jóvenes están recibiendo tanta atención que atrae a más jóvenes al movimiento". Tyrone Scott, encargado de campañas de Young Greens, la rama estudiantil y juvenil del Green Party del Reino Unido, explica en una entrevista que:

Como (activista) verde, el mundo puede parecer un lugar muy sombrío en este momento, pero debemos tener en cuenta que la revolución comienza ahora. Continuaremos saliendo a las calles, ya sea como parte de las Huelgas Escolares por el Clima, Extinction Rebellion o como el Partido Verde, y no descansaremos hasta que los líderes mundiales se comprometan a tomar medidas decisivas para hacer frente a esta emergencia climática global. (Scott, 2020)

A continuación, se presentan algunas de las características más definitorias de estos nuevos movimientos sociales:

a. Marcos conceptuales. Según Díaz Pérez (2020), la activista Greta Thunberg ha propiciado la construcción de un marco y una identidad compartida entre los miembros de Fridays for Future en el cual el agravio es el futuro en riesgo, el "nosotros" es la juventud del planeta con la ayuda de la comunidad científica, "Ios otros" son los líderes políticos del presente y sus aliados en los medios de comunicación tradicionales, el mal a combatir sería la dependencia del petróleo y los combustibles fósiles y la estrategia del movimiento consistiría en hacer huelgas y manifestaciones masivas hasta conseguir que los gobiernos del planeta se comprometan con el cumplimiento de los Acuerdos de París. El desarrollo de marcos conceptuales en torno al movimiento Fridays for Future ha sido especialmente relevante a nivel comunicativo, dada la carencia de encuadres que tradicionalmente ha afectado a los movimientos sociales en defensa del medioambeinte (Lakoff, 2010). Los medios de comunicación y las redes sociales se conforman en su imaginario como una herramienta, a veces a favor de los otros $o$ a veces sus aliados.

b. El mensaje. Existe un mensaje compartido dentro del ecosistema de los movimientos sociales ecologistas: los líderes mundiales están poniendo en riesgo nuestro futuro, pasan a formar parte de la identidad colectiva y los marcos compartidos por los activistas (Díaz Pérez et al., 2021). El discurso de este grupo de activistas se centra en un discurso emocional, con la esperanza como principal emoción activadora, complementada con la rabia o resentimiento hacia aquellos que tienen la culpa de la crisis climática (las grandes corporaciones y las organizaciones como las Naciones Unidas o la Unión Europea), y sus cómplices: los medios de comunicación tradicionales. En general, las reivindicaciones del colectivo están dirigidas a promover cambios profundos, esto es, "no solo políticas públicas a favor del medio ambiente sino cambios culturales de fondo que logren transformar las sociedades" (Paz, 2021).

c. Perfil de los activistas. Los miembros de estos nuevos movimientos sociales son de ideología progresista (ecologistas, feministas, antirracistas) y muestran su compromiso con causas de justicia social y climática (Díaz Pérez, 2020). Como jóvenes, la mayoría tienen entre 16 y 25 años, aunque pueden encontrar apoyos y activos importantes entre activistas de otras generaciones. Con respecto al género, las mujeres son más proclives a participar con asiduidad en protestas como las de Fridays for Future (Noth y Tonzer, 2022). 
Según Adam y Kriesi (2007), la conflictividad y las estrategias represivas del Estado se relacionan con tasas de participación menores, mientras que los niveles de libertad y paz social las maximizan. Los jóvenes están utilizando sus herramientas en la formación y la educación de alto nivel no solo para acceder al conocimiento, sino también para implementarlo en sus rutinas diarias o causas activistas. Estos paradigmas innovadores ofrecen muchas oportunidades para convertirse en activista no solo en el tiempo libre, sino también como parte de sus carreras. Además, no solo están utilizando la educación para mejorar su activismo y capacidad de impacto, sino también para empoderar a otras personas en el proceso, lo que la convierte en una herramienta realmente importante para una solidaridad creciente (Scott, 2020).

d. El colectivo. Este grupo hace un Ilamamiento hacia la solidaridad, ligada a la identidad colectiva y lejos de la llamada me, me, me generation (generación yo, yo, yo) que caracterizó a los millennials. Como explica Alberto Melucci (1996), la identidad colectiva es la que dota de sentido a la participación y ayuda a entender la unidad de acción y el potencial de movilización cuando un movimiento adquiere visibilidad pública. Según Klandermans (2004) las conexiones sociales contribuyen a prolongar la continuidad de los activistas en el movimiento.

e. Sin líderes. Estos movimientos evitan liderazgos ultrapersonalizados o en forma de grandes héroes. Nombres como Greta Thunberg o Vanessa Nakate aparecen más como un ejemplo a seguir, una voz inspiradora, que un liderazgo fuerte del movimiento. El nuevo activismo es cada día más consciente de que no hay necesidad de una organización o movimiento único para generar un cambio social, y que sus esfuerzos pueden pasar desapercibidos. Lo que realmente cuenta es la suma de todos los intentos individuales de justicia climática.

f. La forma de protesta. La estrategia de los nuevos movimientos sociales se centra, principalmente, en dos grandes espacios: las calles y las redes sociales (Díaz Pérez et al., 2021). Si bien es cierto que existen otros métodos que utilizan a menudo, como la reunión con representantes políticos $u$ organizaciones gubernamentales o administrativas para hacer presión para conseguir sus demandas, las manifestaciones y las redes son sus dos espacios de mayor poder, donde son más capaces de trasladar sus demandas y ambiciones. La psicología de la protesta distingue tres motivos distintos para pasar a la acción: la acción instrumental, que tiene la intención de influir en el ambiente político y social; la acción identitaria, motivada por la identificación con el grupo; y la ideológica, que buscaría expresar sus puntos de vista sobre una situación (Della Porta y Diani, 2015). La adecuación de las acciones con las preferencias de los grupos muestra que las manifestaciones y las huelgas son algunas de las maneras preferidas por parte de los jóvenes activistas, que se han promovido mayoritariamente durante los últimos años, pero con la pandemia de la COVID-19 el espacio digital ha tenido que ganar mayor presencia, y esto podría alterar la tendencia de los próximos años.

g. El espacio digital. Las nuevas plataformas virtuales han permitido a grandes organizaciones no solamente la capacidad de conectar con una audiencia global, sino también de dar testimonio de lo ocurrido, generar conciencia con campañas virtuales de concienciación o presión a gobiernos y transnacionales, así como generar espacios de 
debate en línea para el intercambio de prácticas, ideas y contraste de iniciativas y propuestas. Especialmente las redes sociales se han mostrado como una herramienta valiosa en términos de organización y alianzas a nivel global. Existen grandes retos aún en el mundo digital, como el cibercrimen, la infodemia, los debates como los límites de la privacidad y el poder creciente de las grandes compañías tecnológicas, que tienen efectos cada vez más directos en nuestras vidas. Sin embargo, el número de seguidores en Twitter, Facebook e Instagram tienen una correlación positiva y significativa con la movilización, siendo Instagram la red social que mayor participación propicia entre los nuevos movimientos sociales (Díaz Pérez et al., 2021). En el caso de España no encontramos un origen tan participativo como en otros estados, a lo que se suma un carácter menos individualista y más organizacional en Twitter frente al movimiento matriz (Rivas-de-Roca, 2020). Los resultados de Soler i Martí, Ferrer-Pons y Terren (2021) sugieren una relación estrecha y de refuerzo mutuo entre el activismo online y offline, con picos de actividad e interacciones en Twitter que generalmente giran en torno a acciones de protesta en la calle. Las nuevas tecnologías propician la implicación de actores dispersos geográficamente y permiten responder a problemas transnacionales con un rápido repertorio de contención (Díaz Pérez, 2020). Además, reducir la dependencia de los medios de comunicación tradicionales que en muchas veces ignoran las actividades de los activistas o amplifican las narrativas de sus adversarios se ve como un activo a favor de sus causas. Pese a ello, el activismo juvenil ecologista ha conseguido influir con sus protestas y demandas la cobertura informativa de los medios tradicionales, como ocurrió con las principales televisiones españolas durante la Semana de Acción Climática de 2019 (TesoAlonso, 2021).

h. La crisis de la pandemia de la COVID-19. La pandemia de la COVID y su posterior crisis abren algunas posibilidades en los movimientos sociales juveniles de los recientes años. La nueva alarma de la pandemia no solamente es una evidencia más de la emergencia climática, también representa la entrada en escena de una nueva área: la digital. En las sociedades prepandémicas la información ha sido un elemento claro para entender y ejercer el poder social de los movimientos ecologistas. El impacto de la pandemia alrededor del planeta forzó a la mayoría de los países a adaptarse y tomar medidas drásticas para frenar el avance del virus y mitigar sus impactos en la economía y sociedad. A nivel comunicativo, esta crisis sanitaria desplazó otros asuntos de la agenda informativa, como el cambio climático, y las movilizaciones ciudadanas perdieron en 2020 el protagonismo que tuvieron en 2019 (Fernández-Reyes, 2021). Gracias a la estrategia del \#ClimateStrike, el movimiento consiguió mantener una actividad constante en redes sociales, aunque en términos absolutos los números de participación tanto offline como online bajaron cuantiosamente durante abril y septiembre (Díaz Pérez, 2020).

En conclusión, y como muestra Díaz Pérez (2020), existe una alta y significativa correlación positiva entre las tasas de escolarización, el PIB y el acceso a internet con el porcentaje de población movilizada. Las características de estos nuevos movimientos sociales no solamente están conformando un nuevo grupo activista con grandes diferencias respecto a generaciones anteriores, también están conformando un modo de vida nuevo que concilia el activismo con las prácticas diarias de cada uno de sus individuos. En unos años quizás veamos cómo estas características revierten en prácticas sociales consolidadas. 


\section{CONCEPTUALIZACIÓN DEL PROBLEMA}

Con la aparición de algunos líderes como Greta Thunberg, la famosa activista de Suecia, parecía que, por primera vez en mucho tiempo, los adultos escuchaban las demandas de sus jóvenes. Por ejemplo, el secretario general de la ONU, António Guterres, ha respaldado las huelgas escolares, afirmando que: "Mi generación ha fallado en gran medida hasta ahora para preservar tanto la justicia en el mundo como para preservar el planeta. Tengo nietas y quiero que vivan en un planeta habitable. Mi generación tiene una gran responsabilidad; y es su generación la que debe hacernos responsables para asegurarnos de no traicionar el futuro de la humanidad" (Naciones Unidas, 2019). Sin embargo, ¿̇realmente se escuchan las demandas de estos nuevos movimientos sociales, o es solo parte de una estrategia de lavado verde para alcanzar el interés de los más jóvenes? En este escenario, ¿qué deberían hacer los jóvenes activistas? ¿Frenar sus demandas y bloquear su posible instrumentalización de los organismos internacionales? 0 , por el contrario, ¿utilizar esta instrumentalización para lograr mejor sus objetivos?

El origen del término advocacy trap se encuentra en el sindicalismo en artículos como The Advocacy Trap: when legitimacy building inhibits organizational learning (Zuzul \& Edmonson, 2017) o The Advocacy Trap: Transnational Activism and State Power in China (Noakes, 2017). Sin embargo, para este artículo se ha definido el término de la trampa de la incidencia (traducción del inglés advocacy trap) de la forma siguiente:

La trampa de la incidencia es aquella situación donde los jóvenes activistas ecologistas topan con sentimientos de frustración, decepción o desesperación ante su incapacidad de trasladar de forma directa sus demandas a las instituciones que, o bien no toman en serio sus demandas, o bien las utilizan para propósitos distintos de los reclamados por parte de los activistas. La trampa puede resultar en reacciones como el abandono del activismo por parte de los jóvenes o el agotamiento de los movimientos.

Esta trampa, por tanto, consiste en una constante sensación de desesperanza en el corto plazo, que lleva a consecuencias como la pérdida de fe en conseguir trasladar sus propuestas a instituciones o modelar las ambiciones empresariales, conseguir cambios para el bienestar de la comunidad o proteger la biodiversidad ante sus amenazas naturales, pero sobre todo humanas. De esta misma forma, la trampa puede revertir en un impulso positivo en el largo plazo, y servir como impulso de un activismo más fuerte, más preparado y con más recursos y herramientas para enfrentar situaciones de greenwashing, utilización de demandas para fines contradictorios o insistencia en lo propuesto por parte del movimiento social.

\section{CASO DE ESTUDIO}

Para reforzar las tendencias anteriormente revisadas, este estudio se propone analizar mediante una pequeña muestra si la trampa de la incidencia es una situación que afecta a los jóvenes activistas ecologistas en Catalunya. Para este artículo se han realizado una serie de entrevistas con el fin de demostrar si esta situación se percibe por parte del cuerpo activista.

Para comprender las seis entrevistas realizadas a activistas ecologistas de Catalunya hay que tener en cuenta tres cuestiones. En primer lugar, la dificultad que existe al contabilizar los miembros activos de un movimiento. Algunos se encuentran registrados en grupo $u$ organización, pero otros participan de manera más informal, asistiendo a manifestaciones o 
participando en campañas globales o locales. En segundo lugar, es importante destacar que el tiempo y el nivel de desarrollo de conocimientos y capacidades técnicas de la investigadora han presentado una limitación para la investigación. En tercer lugar, cabe destacar que el propósito de este artículo no es elaborar un estudio sobre las características de los jóvenes activistas ecologistas de Catalunya, sino demostrar si la trampa de la incidencia les afecta y existe en sus grupos organizados. Por esta razón, el artículo pretende ser una apertura a una línea de investigación más amplia. Para las entrevistas se han elegido seis activistas con características paritarias de género y con balance de edad, raza, movimiento, educación, lugar de origen y residencia. A continuación, se detallan las características de la muestra por participantes:

- Anna. 20 años, mujer cis caucásica. Milita en Joves Ecosocialistes, Catalunya En Comú y Barcelona en Comú y está cursando un grado universitario tras realizar un grado superior. Originaria y residente de Barcelona.

- Bruna. 20 años, mujer cis caucásica. Milita en Joves Ecosocialistes y está cursando un grado superior de música. Originaria y residente de Barcelona.

- Dan. 22 años, hombre cis caucásico/hispano. Milita en Fridays for Future y es Ingeniero de Materiales. Nació en Barcelona y vive en Alella (Maresme).

- Giovani. 18 años, hombre cis malai. Milita en Fridays for Future Tarragona. Es originario de Filipinas y reside en Tarragona.

- Lucas. 19 años, hombre cis, caucásico. Milita en Joves Ecosocialistes y está cursando un doble grado en Derecho y Ciencia Política en la Universitat Autònoma de Barcelona. Originario y residente de Sitges (Barcelona).

- Nessa. 24 años, mujer trans, caucásica. Milita en Sororitrans y luchas ecologistas y del colectivo LGBTI. Tiene estudios de máster en Derecho Internacional. Originaria de Alpicat residiendo en Barcelona.

Para inferir si estas personas activistas han experimentado la trampa de la incidencia, se ha elegido la técnica de la entrevista semiestructurada. La razón por la que se ha acudido a esta técnica es por la voluntad de extraer información cualitativa centrada en la percepción individual de los activistas, no en los datos más estadísticos que podrían habernos dado encuestas $\mathrm{u}$ otros métodos cuantitativos. Esta investigación busca explorar los efectos de la trampa de la incidencia en los jóvenes activistas ecologistas de Catalunya, para comprender cómo les afecta a nivel individual y de grupo, y los sentimientos que derivan de esta propuesta conceptual. Este análisis preliminar quiere servir como base para futuras investigaciones al respecto.

Para la entrevista semiestructurada se han elaborado seis preguntas iniciales, acompañadas por una intervención inicial donde se explica la trampa de la incidencia a los activistas, para llegar a las conclusiones que se mostrarán en el capítulo siguiente. La Tabla 1 recoge este cuestionario.

Tabla 1

Cuestionario utilizado para las entrevistas semiestructuradas 


\begin{tabular}{|c|l|}
\hline Número de la pregunta & \multicolumn{1}{|c|}{ Texto de la pregunta } \\
\hline $\begin{array}{c}\text { Introducción (explicación } \\
\text { del concepto de la } \\
\text { trampa de la incidencia) }\end{array}$ & $\begin{array}{l}\text { Situación donde los jóvenes activistas ecologistas topan con sentimientos de } \\
\text { frustración, decepción o desesperación ante su incapacidad de trasladar de } \\
\text { forma directa sus demandas a las instituciones que, o bien no toman en serio } \\
\text { por parte de los activistas. La trampa puede resultar en reacciones como el } \\
\text { abandono del activismo por parte de los jóvenes o el agotamiento de los } \\
\text { movimientos. }\end{array}$ \\
\hline Pregunta 1 & $\begin{array}{l}\text { ¿Cuál es el mayor reto, según tu opinión, del activismo juvenil ecologista en } \\
\text { Catalunya? ¿Cuáles crees que son sus principales causas? }\end{array}$ \\
\hline Pregunta 2 & $\begin{array}{l}\text { Como activista, ¿has sentido en alguna ocasión una situación similar a la } \\
\text { trampa de la incidencia? }\end{array}$ \\
\hline Pregunta 3 & $\begin{array}{l}\text { ¿Cómo te sientes cuando esta situación sucede? ¿Cuál es tu sentimiento } \\
\text { dominante? }\end{array}$ \\
\hline Pregunta 4 & $\begin{array}{l}\text { ¿Crees que tus compañerxs comparten este sentimiento? ¿Crees que es una } \\
\text { cuestión de cómo son las personas o que es un sentimiento generalizado? }\end{array}$ \\
\hline Pregunta 5 & $\begin{array}{l}\text { ¿Cuándo te encuentras en esta situación, ¿cómo reaccionas? ¿Qué haces y } \\
\text { qué noces? ¿Te gustaría actuar de un modo distinto, pero no lo haces por } \\
\text { algún motivo? }\end{array}$ \\
\hline ¿Crees que esta situación podría revertirse? ¿Qué sería necesario para que \\
esto ocurriera?
\end{tabular}

Una vez realizadas las entrevistas se contrastaron sus resultados de forma comparada, y se extrajeron las conclusiones que se pueden leer en el siguiente apartado.

\section{RESULTADOS}

Las entrevistas a los jóvenes activistas ecologistas brindaron luz a algunas de las cuestiones contempladas en la definición de la trampa de la incidencia. Este apartado recoge algunas de las más relevantes.

En primer lugar, encontramos que todas las activistas ${ }^{1}$ han manifestado vivir o haber vivido una situación similar a la trampa de la incidencia. En particular, Anna expone que ella ha vivido la trampa en diferentes partes, pero con la pandemia sus efectos han aumentado por la falta de contacto y el aumento de la dificultad de organizarse, hecho que le causó una total incapacidad de trasladar sus demandas. Dan afirma que cree que existe la trampa de la incidencia, pero que no cree que sea un problema exógeno del sistema, sino que estaría presente en todas las instituciones capitalistas. Para este, el techo se representa en la dificultad de incidir en la manera como se hacen las cosas, donde no solo las demandas cuestan de ser trasladas por su naturaleza, sino que también es difícil la interacción con aquellos sectores o personas representantes de ellas, con poca disposición al cambio. Algunos optimistas apuntan que el movimiento ecologista tiene una fuerza impulsora del cambio, puesto que está respaldada por la ciencia y por los grandes convenios internacionales. Sin

\footnotetext{
${ }^{1}$ En la exposición de resultados se ha alternado el uso del masculino y el femenino como genéricos para promover un uso inclusivo del lenguaje lo más respetuoso posible al hacer referencia a las personas entrevistadas.
} 
embargo, otros se desesperan ante la falta de acción por la falta de tiempo. Las causas de ello radican, según Giovani, en el distanciamiento que existe entre instituciones y gobernantes. "Entre instituciones y activismo existe mucha distancia, y se nota sobre todo en las demandas y reivindicaciones. Por parte de los activistas, se ciñen al reglamento y desde las administraciones públicas llegan distorsionadas e, incluso cuando se pueden transformar en medidas, resultan insuficientes o escasas", explica el entrevistado.

Cuando evalúan el escenario con el que se encuentra el activismo ecologista en Catalunya, la mayoría valora que los principales retos son, de un lado, organizacionales $y$, de otro, de sistema. Anna, por ejemplo, destaca la necesidad de formar redes y centralizar las demandas. Una mayor coordinación y diálogo entre las organizaciones podría ayudar a trasladar de una forma más efectiva y potente los mensajes y demandas que muchos colectivos ya comparten. "Tenemos que hacer pedagogía y a la vez ser altavoz", expone Anna. Por otro lado, Lucas señala que, por la tradición del movimiento, uno de los mayores desafíos es relacionar la causa climática con los demás retos, como el clasismo o el racismo persistente en nuestras sociedades. En este sentido, la mayoría de las activistas coincidieron en la falta de medidas y compromiso para canalizar las demandas de colectivos sociales a las propuestas legislativas o procedimientos públicos. "No caer en consignas y salir del mensaje posmoderno" es lo que Bruna considera más relevante, donde afirma que, si bien los eslóganes bien pensados y populares pueden ayudar a concienciar y sumar personas al movimiento, las demandas deben ir más allá y transformarse en contrapropuestas y acciones más contundentes.

En cuanto a las emociones, la mayoría de las activistas afirmaron sentir frustración, impotencia, cansancio e inutilidad, entre otras. Todas ellas son de carácter negativo o pesimista, que conducen a pensar que su estado de ánimo se puede ver debilitado por estas barreras sentimentales. Haría falta una futura investigación para abordar la posible correlación existente entre el activismo juvenil ecologista en Catalunya y sus efectos emocionales en la continuidad del movimiento.

Cuando se les preguntó por los casos o ejemplos en los que consideran que se da la trampa de la incidencia, sorprendió encontrar una gran diversidad de situaciones. Desde reuniones con policy makers, a manifestaciones en las calles o movilizaciones masivas, fueron apuntadas como situaciones que, cuando encontraban barreras u obstáculos por parte de la administración o las instituciones, incluso por parte de grupos reaccionarios al movimiento ecologista, les producían todas estas emociones de frustración, insatisfacción, cansancio e inutilidad.

Un dato revelador es la sensación de desgaste o agotamiento que todas las participantes apuntaron, donde la trampa de la incidencia les afecta negativamente a su predisposición a seguir con su activismo ecologista. Muchas de las activistas han mostrado el desgaste personal que supone la lucha constante, el hacer del activismo un modo de vida o una campaña permanente y cómo, en caso de fracaso, es fácil asumir la derrota como un hecho personal. Para ello, existen dos reacciones muy marcadas: encontrar apoyo en el grupo, compartiendo la experiencia con los demás miembros, buscando apoyo en compartir y valorar conjuntamente lo que ha fallado y cómo se puede mejorar de cara a futuras situaciones; o evadirse, distanciarse un poco del activismo durante un corto período de tiempo para poder evaluar con perspectiva lo que ha pasado y aprender de ello. Es llamativa la gran presión a la que se 
someten las personas entrevistadas, hecho que pudiera ser debido a una tendencia de las personas autoexigentes o muy perfeccionistas a participar más de entornos activistas.

La reacción o consecuencia de esta situación de frustración es la evaluación. Todos los activistas han afirmado que este paso es siempre un factor clave para procesar la emoción, bien sea en grupo o individualmente, en el mismo momento en que sucede o al cabo de unas semanas. Por ejemplo, Anna explica: "No es muy activista, pero prefiero dar un paso atrás, mirarlo desde fuera y decir: vamos a ver qué ha pasado, vamos a ver qué hay y mirarlo con perspectiva. Si no está funcionando intento salir para volver a entrar". En cambio, otras como Bruna intentan repetir la tarea de otra forma, para no darse por rendida antes de explorar todas las posibles estrategias. Para Nessa, es imprescindible tomar distancia con el resultado a partir del tiempo. El recurso de Lucas surge del colectivo, donde se apoya en consejos y palabras de su entorno para reconducir su visión y "volver a colocar aquello que ha sido rechazado o por lo que no me han hecho caso en las instituciones". Por último, Dan explica cómo finalmente continúa siempre contribuyendo por su creencia en la causa y su sentido de responsabilidad de parar lo que es inevitable que nos afecte a todas (el cambio climático). Si bien por un lado lo pasa mal con malas noticias que le quitan ganas y energía, hecho que le provoca tristeza, también se reconforta en lo logrado hasta el momento y las cuestiones más personales del activismo, como las amistades.

Finalmente, en la pregunta sobre la posibilidad de revertir la trampa de la incidencia o eliminarla, las personas entrevistadas se mostraron especialmente escépticas. Algunos apuntaron a la muy difícil probabilidad de un cambio de paradigma, debido a lo consolidadas que se encuentran la mayoría de las instituciones del statu quo. Anna, por ejemplo, explica que, según ella, esta situación forma parte de un sistema que nunca se revertirá porque la pirámide de poder siempre estará de la misma forma. Nessa explica que ve muy difícil la desaparición de la trampa debido al reto sistémico que supone, y remarca sobre todo el hecho de que son unas demandas que no son nuevas, y hace muchos años que se ignoran institucionalmente. Otros, como Dan, apuntan a que, si el movimiento deviene mayoritario, existe una posibilidad, aunque baja, de conseguirlo. Para Lucas, la solución se encuentra en el "entrismo" (estrategia de los movimientos sociales para "entrar" en las instituciones para defender desde allí sus causas activistas) y la institucionalización, jugando con o en el sistema para introducir las demandas en la agenda. También hay voces optimistas, como las de Bruna y Giovani, quienes coinciden en que la capacidad de dialogar y cambiar la forma en la que las personas piensan puede ofrecer, si no un cambio general de paradigma, avances hacia un sistema más participativo, abierto y dispuesto a escuchar.

\section{CONCLUSIÓN}

El activismo juvenil ecologista de hoy parece estar vinculado a una cosmovisión más amplia, solidaria y a un conjunto de valores ligados a la idea de igualdad para la prosperidad, lejos del enfoque individualista de anteriores movimientos sociales, construido en torno a la figura de un líder u organización individual. A pesar de que esto aparece como un factor o punto aleatorio en la evolución de los nuevos movimientos sociales (NMS), sus efectos podrían ser mayores de lo que esperamos.

La ruptura con la dinámica individualista del sistema político, económico y social que gobierna el mundo de hoy representa otro factor de ruptura con la dinámica consolidada del 
capitalismo. La aparición de jóvenes más centrados en el propósito de sus acciones que no en aparecer en la portada de un periódico o tener un eco personal, lejos del deseo de encarnar en su persona todo un movimiento, representan un gran avance en materia de activismo y contribuyen a la creación de nuevas dinámicas más plurales, inclusivas y orientadas a la prosperidad colectiva (Díaz Pérez et al., 2021).

En este artículo se han analizado cualitativamente los efectos que la "trampa de la incidencia" tiene sobre los jóvenes activistas ecologistas de Catalunya. El artículo recoge cómo los activistas se encuentran con sentimientos de frustración, decepción y desesperación ante la incapacidad de trasladar de forma directa sus demandas a las instituciones, y cómo esto tiene a su vez consecuencias indeseadas como la pérdida de fe en la lucha del movimiento, la posibilidad de cambio o el sistema en general. Entre las seis activistas encuestadas, se han mostrado los efectos cualitativos que tiene la trampa de la incidencia en el día a día del activismo juvenil ecologista de los llamados nuevos movimientos sociales: la frustración, desesperación o impotencia que produce la trampa generan un desgaste personal y emocional de los activistas que, si bien a algunos casos les puede servir como una forma de mejorar o reconducir las estrategias, puede derivar en el abandono del movimiento o del activismo temporal o definitivamente.

El objetivo de esta investigación no reside en demostrar la existencia de la trampa de la incidencia a gran escala, sino en explorar la utilidad de esta definición para indagar en los efectos que tienen los bloqueos u obstáculos del sistema ante la transición de las demandas entre la sociedad civil y las instituciones sobre los jóvenes activistas. Este análisis se limita al ámbito de Catalunya, futuros estudios pueden expandirse a otros contextos y utilizando otras técnicas. Es importante destacar que, por la falta de recursos, este artículo sólo ha podido centrarse en una pequeña muestra y tiene carácter preliminar, pero que una investigación similar podría replicarse con una muestra más grande o en un formato que permitiera establecer comparaciones entre regiones o países.

También se abre la puerta a una futura investigación más en detalle de las causas de estos obstáculos, así como una propuesta de mejoras para que la trampa de la incidencia se reduzca o desaparezca en el activismo juvenil ecologista. Algunas de las líneas de investigación que derivan del caso estudiado serían matizar el fenómeno de la trampa de la incidencia en territorios donde jóvenes sufren las consecuencias más directas del cambio climático, por ejemplo, del sur global donde existen zonas de sacrificio, asesinatos a líderes y activistas socioambientales, extractivismo y efectos severos del cambio climático, entre otros. La intención de seguir la investigación comparando este fenómeno en otros países, e incluir el enfoque decolonial en el rol de activista socioambiental o socioecológico europeo y occidental está presente en este primer análisis.

Los movimientos sociales supusieron un cambio en la visibilización de la crisis climática en los años anteriores a la pandemia. El activismo es fundamental para poner el problema climático en las agendas mediática y política. La trampa de la incidencia puede ser una barrera en el desarrollo de estos movimientos tan necesarios en un contexto de crisis climática. Así, superar la trampa de la incidencia puede ser un avance hacia una gobernanza más participativa, más conectada y efectiva para dar respuesta a los retos climáticos que vendrán en los próximos años. 


\section{Referencias}

Adam, S., y Kriesi, H. (2007). El enfoque de redes. En P. A. Sabatier (Ed.) Teorías del proceso de las políticas públicas, (pp. 139-348).

Demaria, F., Schneider, F., Sekulova, F., \& Martinez-Alier, J. (2013). What is degrowth? From an activist slogan to a social movement. Environmental Values, 22(2), 191-215. https://doi.org/10.3197/096327113×13581561725194

Della Porta, D., \& Diani, M. (Eds.) (2015). The Oxford handbook of social movements. Oxford University Press. https://doi.org/10.1093/oxfordhb/9780199678402.001.0001

Díaz Pérez, S., Soler-i-Martí, R., \& Ferrer Fons, M. (2021). Del mito global a la movilización local: Creación y resonancia del marco Greta Thunberg. Comunicar, 29(68), 35-45. https://doi.org/10.3916/c68-2021-03

Díaz Pérez, S. (2020). El futuro en llamas: Greta Thunberg y Fridays for Future. Beers and Politics. https://bit.ly/3oRxymt

Fernández-Reyes, R. (2021). La comunicación de la crisis climática en tiempos de crisis sanitaria. En D. Rodrigo-Cano, R. Mancinas-Chávez, y R. Fernández-Reyes (Eds.), La comunicación del cambio climático, una herramienta ante el gran desafío (pp. 124-164). Dykinson. https://bit.ly/3EQcQJo

Fisher, D.R. (2019). The broader importance of \#FridaysForFuture. Nature Climate Change, 9, 430-431. https://doi.org/10.1038/s41558-019-0484-y

Flynn, C., Yamasumi, E., Fischer, S., Snow, D., Grant, Z., y Kirby, M. (2021). Peoples' Climate Vote. UNDP y Universidad de Oxford. https://bit.ly/3s4LbAR

Fridays for Future (n.d.). Strike statistics. Fridays for Future. Consultado el 9 de diciembre de 2021: https://bit.ly/3dwzrif

Hernández Ballesteros, M. L. (2020). Movilizaciones ecologistas juveniles y desarrollo alternativo. Estudio de caso: Fridays For Future Madrid (2019-2020). [Trabajo de Final de Máster]. Universidad Pontificia de Comillas.https://bit.ly/320x1WK

Jenkins, H., Shresthova, S., Gamber-Thompson, L., Kligler-Vilenchik, N., y Zimmerman, A. (2018). By any media necessary: The new youth activism. European Journal of Communication, 34(5), 571-571. https://doi.org/10.1177/0267323119876117a

Juris, J., Pereira, I., y Feixa, C. (2012). La globalización alternativa y los 'novísimos' movimientos sociales. Revista del Centro de Investigación de la Universidad la Salle, 10(37), 23-39. https://doi.org/10.26457/recein.v10i37.107

von Kardorff, E. (2019). Castells (1996): The Rise of the Network Society. En Holzer B., Stegbauer C. (Eds.) Schlüsselwerke der Netzwerkforschung (pp. 105-109). Springer. https://doi.org/10.1007/978-3658-21742-6_25

Klandermans, B. (2004). The demand and supply of participation: Social-psychological correlates of participation in social movements. En D. A. Snow, S. A. Soule, y H. Kriesi (Eds.), The Blackwell companion to social movements (pp. 360-379). Blackwell Publishing. https://doi.org/10.1002/9780470999103.ch16

Kirshner, B. (2015). Youth activism in an era of education inequality. NYU Press. https://doi.org/10.18574/nyu/9781479861316.001.0001

Keck, M. E., y Sikkink, K. (2014). Activists beyond borders: Advocacy networks in international politics. Cornell University Press. https://doi.org/10.7591/9780801471292

Kühne, R. W. (2019, 2 de septiembre). Climate change: the science behind Greta Thunberg and Fridays for future. OSFPreprints. https://doi.org/10.31219/osf.io/2n6kj

Lakoff, G. (2010). Why it matters how we frame the environment. Environmental Communication, 4(1), 70-81. https://doi.org/10.1080/17524030903529749 
Melucci, A. (1996). Youth, time and social movements. Young, 4(2), 3-14. https://doi.org/10.1177/110330889600400202

Noakes, S. (2017). The advocacy trap: Transnational activism and state power in China. Manchester University Press. https://doi.org/10.7228/manchester/9781526119476.001.0001

Noth, F., \& Tonzer, L. (2022). Understanding climate activism: Who participates in climate marches such as "Fridays for Future" and what can we learn from it?. Energy Research \& Social Science, 84, 102360. https://doi.org/10.1016/j.erss.2021.102360

Naciones Unidas (2019, 21 de septiembre). Jóvenes de todo el mundo exigen a los políticos acción contra el cambio climático. Noticias ONU. https://bit.ly/3pNF4hQ

O'brien, K., Selboe, E., y Hayward, B. M. (2018). Exploring youth activism on climate change. Ecology and Society, 23(3), 42. https://doi.org/10.5751/es-10287-230342

Ospina Peralta, P., Idrobo Hidalgo, M. A., \& Ospina Peralta, A. T. (2019). ¿Por qué luchar? Motivaciones, organización y estrategias de la militancia juvenil radical en el Siglo XXI. Grupo Faro. https://bit.ly/31LtuvF

Paz, F. (2021). Lo personal es colectivo: jóvenes y medio ambiente. Beers and Politics, 15, 52-55. https://bit.ly/3oSoSwk

Rivas-de-Roca, R. (2020). La configuración del fenómeno ecologista FridaysForFuture como proceso de opinión pública digital en España. Dígitos. Revista de Comunicación Digital, 6, 79-100. https://doi.org/10.7203/rd.v1i6.162

Sabherwal, A., Ballew, M. T., van Der Linden, S., Gustafson, A., Goldberg, M. H., Maibach, E. W., ... y Leiserowitz, A. (2021). The Greta Thunberg Effect: Familiarity with Greta Thunberg predicts intentions to engage in climate activism in the United States. Journal of Applied Social Psychology, 51(4), 321-333. https://doi.org/10.1111/jasp.12737

Scott, T. (2020, 14 de enero). An unstoppable wave of youth activism. Green World. https://bit.ly/31gHAAK

Soler i Martí, R., Ferrer-Fons, M., y Terren, L. (2020). The interdependency of online and offline activism: A case study of Fridays For Future-Barcelona in the context of the COVID-19 lockdown. Hipertext.net, 21, 105-114. https://doi.org/10.31009/hipertext.net.2020.i21.09

Teso-Alonso, G. (2021). Los movimientos sociales juveniles ante la emergencia climática y la comunicación del cambio climático. En D. Rodrigo-Cano, R. Mancinas-Chávez, y R. FernándezReyes (Eds.), La comunicación del cambio climático, una herramienta ante el gran desafío (pp. 54-120). Dykinson. https://bit.ly/3EQcQJo

Tilly, C. (2006). Los movimientos sociales entran en el siglo veintiuno. Política y sociedad, 42(2), 11-35.

Wahlström, M., Sommer, M., Kocyba, P., de Vydt, M., De Moor, J., Davies, S., ... y Buzogany, A. (2019). Protest for a future: Composition, mobilization and motives of the participants in Fridays For Future climate protests on 15 March 2019 in 13 European cities. Keele University Research Repository. https://bit.ly/3y8cvz6

Wallis, H., y Loy, L. S. (2021). What drives pro-environmental activism of young people? A survey study on the Fridays for Future movement. Journal of Environmental Psychology, 74, 101581. https://doi.org/10.1016/j.jenvp.2021.101581

Zuzul, T., \& Edmondson, A. C. (2017). The advocacy trap: When legitimacy building inhibits organizational learning. Academy of Management Discoveries, 3(3), 302-321. https://doi.org/10.5465/amd.2015.0086

\section{Semblanza de la autora}

Ariadna Romans i Torrent es politóloga por la Universitat Pompeu Fabra y estudiante de Filosofía en la Universitat de Barcelona. Actualmente trabaja en ideograma como consultora 
de comunicación política y es miembro de la comisión mixta de la Cátedra ideograma-UPF. También es la gestora actual de la Secció de Feminismes del Ateneu Barcelonès. Fue presidenta de la asociación juvenil deba-t.org y miembro del programa Young Mediterranean Voices, entre otros proyectos sociales. Ha trabajado para la Comisión Europea y Open Society Foundations. Sus áreas de investigación son las relaciones internacionales, el desarrollo sostenible, la transición ecológica y la ética digital. 